\title{
WHAT DO WE DO WHEN MODELS DON'T FIT? ON MODEL ATMOSPHERES AND REAL STELLAR SPECTRA
}

\author{
BENGT GUSTAFSSON \\ Uppsala Astronomical Observatory \\ Box 515, S-751 20 Uppsala, Sweden \\ (Bengt.Gustafsson@astro.uu.se)
}

\begin{abstract}
Developments in the modelling of stellar atmospheres and results from the confrontation between calculated and observed fluxes and spectra are discussed. It is argued that, although impressive advances in the study of O-type, WR and cool star spectra have recently occurred, significant improvements should be possible with already existing methods in the analysis of, e.g., solar-type stars.
\end{abstract}

\section{Introduction}

The art of interpreting stellar spectra and fluxes relies on models of the flux-emitting layers, the stellar atmospheres. However, these models are never perfect, and often unsatisfactory. The question in the title is therefore relevant in almost all analyses of stellar radiation. An answer to this question, as given in practical work, is often the following: First, we change the fundamental parameters of the models to get a good fit. If such a fit is obtained with a certain parameter set, these specific parameter values are dubbed "determinations" of, e.g., effective temperature or abundances. If the variation of parameters does not resolve the mis-match, we question the input physics, such as $g f$ values or opacities. Also, the observations may be scrutinised. If these efforts cannot explain away our problems we blame the basic assumptions of the models, like LTE or hydrostatic equilibrium. This procedure may well be adequate in many cases, but - in spite the welltested scientific prescription to always first try the simplest explanations, which goes back to medieval theology ("Occam's razor") - may also lead us astray.

T.R. Bedding et al. (eds.),

Fundamental Stellar Properties: The Interaction between Observation and Theory, 261-276.

(C) 1997 IAU. Printed in the Netherlands. 
There is also a reverse, theoretical and more critical tradition in this field of research. Here, attempts to improve the internal physical consistency of the models are in focus. If this can be achieved (if not, the discussion may turn rather frustrating for practitioners interested in applications and results more than in philosophical principles), the predicted spectra of the new models are compared with the old ones and observations are used to support the claim that the new models are indeed more realistic.

However, these two ways towards a better understanding of stellar spectra and stellar atmospheres are rarely so simple and the difference between them not often so clear-cut. Instead, practice in this field today is developing towards mixed approaches, with close interaction between the two different ways of procedure. This partly reflects the methodological advances in the last decades both in observations and in theory. On the observational side, the enormous improvement in the possibility of getting high S/N spectral data, even at high spectral resolution and extending from the extreme ultraviolet to the far infrared spectral region, has enabled studies of fundamentally new aspects of stellar atmospheres and to check models to an extent far beyond what could be contemplated before. On the theoretical side, new numerical methods now make it possible to abstain from some, or even most of the assumptions behind classical model atmospheres, such as LTE or mixing-length convection. Also as regards basic atomic and molecular data the advance is great, which has put both direct interpretation of stellar spectral line measurements and the modelling of the indirect effects of line blanketing on a much safer ground.

So, today stellar spectroscopic analysis is a field with close and intricate interaction between the two approaches discussed above. This brings new progress and new complications. Sometimes theoretical and more empirical approaches are intertwined so intimately that it is difficult to see how new evidence would affect the conclusions of a study. Also, the great number of different observational criteria that are accessible today for an object may tempt to compromises in model fits or model construction that can be hard to describe or reproduce. In the following some examples will be given of such complex situations, as well as more classical confrontation between the two approaches. I shall start with a presentation of a highly successful fit of standard models, then turn to examples of the great progress in recent years, mainly from theoretical modelling of hot stars, then go to the other extreme in the HR diagram, the cool stars, for which significant advances have recently been made due to improvements in molecular data, and finally end up with stars of intermediate temperatures for which the situation at present seems more frustrating.

My comments are not meant to be comprehensive - the reader is advised to consult the conference proceedings edited by Crivellari et al. (1991) 
and Strassmeier and Linsky (1996) for reviews on progress in atmosphere modelling; see also Gustafsson and Jørgensen (1994) as regards models for late-type stars. Reference to reviews on more specific topics will be made below.

\section{Vega: Success of classical models (?)}

The classical model atmospheres are based the assumptions of plane-parallel stratification, hydrostatic and radiative equilibrium (if need be modified by mixing-length convection) and local thermodynamic equilibrium (LTE). An extensive grid of such models, with detailed consideration of line blanketing, has been produced by Bob Kurucz, and such models were compared in detail with observations of the standard star Vega (AOV) by Castelli and Kurucz (1994). They found that models with $T_{\text {eff }}$ in the interval 9550$9650 \mathrm{~K}$ and logarithmic gravity of at least 3.95 (the exact values depending on $E(B-V)$ and the helium abundance) give the optimal fit to observations. With reddening and He abundance known the observed visual fluxes would enable a determination of $T_{\text {eff }}$ to $50 \mathrm{~K}(0.5 \%)$ and the $\mathrm{H} \gamma$ profile a determination of $\log g$ to 0.05 dex. In the ultraviolet below $200 \mathrm{~nm}$ the computed flux is higher than the observed by typically $4 \%$, and other localised differences appear, probably caused by errors in, or totally missing strong lines. In the visual, the agreement is excellent within the small observational errors $(<1 \%)$, while in the infrared the observed flux is higher than the calculated (e.g., by $3 \%$ at $1040 \mathrm{~nm}$ ). Also, the observed Paschen lines are much broader than the computed ones; the reason for this needs further exploration, but may well turn out to be trivial.

The excellent agreement found by Castelli and Kurucz certainly lends credibility to the validity of the basic assumptions behind classical models. These assumptions may be valid in this case because the atmosphere is not convective and the gravity is high enough to keep the atmosphere well stratified. Certainly, departures from LTE are significant for atoms like Fe, Ba and N (Gigas (1986), Gigas (1988), Takeda (1992), Lemke and Venn (1996), Rentzsch-Holm (1996) and references therein) and these effects, mainly reflecting the hot non-local radiative field in the ultraviolet, must be considered in abundance analysis. Yet, the basic photospheric structure is probably not very much affected in the continuum flux forming layers (cf. Frandsen (1974)). However, models with a relatively complete nonLTE blanketing should be calculated, and compared to corresponding LTE models before one can be sure that the neglect of departures from LTE in the model atmospheres is insignificant. One should also note that Vega has been found to be rapidly rotating but seen pole-on (Gulliver et al. (1994)) which means that $T_{\text {eff }}$ and $\log g$ vary considerably across its surface. 


\section{The virtue of handling radiation properly}

Very impressive progress has recently been made in the analyses for hot stars, as reviewed by John Hillier in these Proceedings. On the theoretical side this is due essentially to three different developments:

Unified models of atmospheres with radiatively driven winds have been developed, in particular by the Munich group (cf. Pauldrach et al. (1993) for a review). This progress will be further illustrated below.

Major advances in techniques for solving radiative transfer problems for complex atoms now make it possible to calculate non-LTE model atmospheres with consideration of all important atomic and molecular transitions. The Accelerated Lambda Iteration (ALI) method, based on the method of Scharmer (1981), has been developed and speeded up, and combined with the statistical approach of Anderson (1989) to handle complex atoms with opacity sampling (Anderson (1991), Dreizler and Werner (1993), and references cited therein). An alternative method is the hybrid method by Hubeny and Lanz (1995), combining the advantage (global convergence) of the classical Complete Linearization Method of Auer and Mihalas with the great speed and flexibility of the ALI. Hubeny and Lanz also develop a statistical treatment of the complex atoms, inspired by Anderson (1989). These major advances are applicable for stars in all parts of the HR diagram, but as yet, they have mainly been applied for the hotter stars. A major remaining problem is the lack of basic physics data, in particular cross sections for inelastic collisions with electrons, atoms and (for the coolest stars) molecules. Another basic restriction is that complete redistribution is assumed within the transitions. If partial redistribution should also be allowed for, further methodological development will be important. In this case, smaller radiative losses are expected from strong lines for both static and dynamic atmospheres, in particular in low-density atmospheric regions (cf. Huenerth and Ulmschneider (1995), Avrett (1996)).

A third area where major improvements have taken place during the latest decade is in atomic absorption data. Thus, the Opacity Project (Seaton et al. (1994)) and the impressive line lists calculated by Kurucz (1995) and other atomic data bases (e.g., Piskunov et al. (1995), Bell et al. (1994)) have enabled a much more realistic treatment of line blanketing.

These advances have especially been applied in the modelling of O-type stars and Wolf-Rayet stars. For the O-type stars the Munich group has constructed spherically extended NLTE-models where the photosphere and the radiation-driven wind are unified in a self-consistent way (Gabler et al. (1989)). The models are successful in reproducing a number of important observational phenomena - e.g. the long standing "Zanstra-discrepancy" in the different temperature estimates from $\mathrm{H}$ and He II lines. Gabler et 
al. (1992) also demonstrated that the increased EUV flux in these models, as compared with plane-parallel ones, make massive $\mathrm{O}$ stars possible candidates for providing enough nebular excitation for the He II emission from high-excitation H II regions. For central stars in planetary nebulae the EUV flux is, however, still higher than predicted in a number of cases, which Gabler et al. (1991) have ascribed to shock generation of extra EUV photons.

Pauldrach et al. (1994) studied the UV spectra of winds of hot luminous stars with a very detailed non-LTE treatment of most important ionization stages. Photospheric line blocking was considered but not completely selfconsistently, and with an approximate calculation of the shock emission. Higher mass loss rates resulted than with previous standard models, and a generally very good agreement between observed and calculated spectra was obtained for $\zeta$ Pup and Melnick 42 - the brightest star in the LMC.

Schaerer and Schmutz (1994) showed, in their first line-blanketed models of spherically expanding atmospheres of hot stars (with simultaneous solution of the equations of motion, the non-LTE problem of $\mathrm{H}$ and $\mathrm{He}$ and radiative transfer in the line-blanketed atmosphere), that even "purely" photospheric lines, on which spectroscopic determinations of stellar parameters are based, are strongly affected by the velocity field in the transition zone between the photosphere and the wind, and that this does not only depend on the mass loss rate but on the velocity structure of the wind. Problems still remain in comparisons with observations, e.g. for $\zeta$ Pup, for spectral lines dependent on the wind structure.

For O-type subdwarfs (sdO stars), which are immediate progenitors to the white dwarfs, fully line-blanketed models have recently been constructed by Haas et al. (1996), using the method of Dreizler and Werner (1993). Haas et al. included a detailed study of the line blanketing effects of iron-group elements but found that the effects on the main optical diagnostics, lines of $\mathrm{H}$ and $\mathrm{He}$ and other light elements, are relatively small.

For Wolf-Rayet stars the so-called standard model - based on the assumption of a spherically symmetric homogeneous expanding atmosphere with a stationary flow - has been elaborated with detailed non-LTE model atmospheres for several elements, and, which is found important, with proper treatment of the frequency redistribution of line photons by electron scattering (cf. reviews by Hamann (1995), Hillier (1995)).

In analyses of observed WR spectra the parameters of the standard model are estimated with accuracies of typically $10 \%$ for $T_{\text {eff }}$ and the velocity parameter, of $20 \%$ for the radius parameter and about $30 \%$ for the mass-loss rate.

The different shortcomings of the standard model, such as predicted too strong P-Cygni absorption of He I lines, or too strong electron scat- 
tering wings, or the observational indications of polarisation, suggesting departures from spherical symmetry, and line-profile variations, suggesting wind inhomogeneities, and strong X-ray fluxes from many stars, are further commented on by Hillier ((1995)).

An important and quite difficult step to take for the hot stars is modelling of clumps or other departures from spherical symmetry. It is possible that this step will be needed in order to decrease the errors in fundamental parameters much below those of today.

A basic link is still missing for the WR stars: the velocity structure of the wind is not calculated from first principles. Also, not the least for these stars, departures from spherical symmetry are known to occur, and these require further detailed exploration.

\section{Cool giant opacities: just a beginning}

The progress in atomic line data is matched for cool stars by corresponding improvements for molecules. Very extensive calculations and compilations of molecular line data have recently been made (cf. Gustafsson and Jørgensen (1994) and Jørgensen (1997) for recent reviews) and these have led to a much more realistic treatment of line blanketing as well as improved calculations of these heavy line-blocked spectra and colours. In spite of these achievements, still more improvements are needed (cf., e.g., Gustafsson (1995) for a summary of current opacity needs for cool star modelling).

For the $\mathrm{M}$ and $\mathrm{C}$ stars only LTE models with mixing-length convection exist until now. The most realistic model grid for non-Mira high-luminosity M stars is the opacity sampling grid of Plez et al. (1992). When comparing these models to observed fluxes these authors found a fair agreement with the IR fluxes of three $M$ giants with $T_{\text {eff }}$ in the interval $3200 \mathrm{~K}-4000 \mathrm{~K}$. The visual fluxes are predicted to be too strong, probably because of missing opacity. In the visual and near IR, the model TiO bands come out too strong, seemingly with a discrepancy increasing with decreasing temperature. Thus for $\mathrm{g}$ Her $\left(T_{\text {eff }}=3235 \mathrm{~K}\right)$ they found the reduction needed of the band oscillator strengths to be more than a factor of two. A possibility that the mismatch reflects non-LTE effects, over-ionization in Ti, keeping the abundance of $\mathrm{TiO}$ molecules below its thermal values, should be investigated further, but may not seem very probable in view of the results reported by Allard et al. (1994). Considerably revised measured band oscillator strengths of $\mathrm{TiO}$ have recently been introduced in synthetic spectra by Plez (cf. Bessell et al. (1997)) and these tend to lead to a much better agreement.

Plez et al. (1992) also found a satisfactory agreement between calculated and observed $V, R, I, J, K$, and $L$ colours. A new much more exten- 
sive comparison between calculated and observed colour-colour relations and temperature-colour relations for A-M stars is being made by Bessell, Castelli and Plez (1997)).

Grids of blanketed LTE-model atmospheres of cool carbon stars have been published most recently by Jørgensen et al. (1992). These models are spherically symmetric and include polyatomic absorption by $\mathrm{HCN}, \mathrm{C}_{2} \mathrm{H}_{2}$, shown to have very great structural effects by Eriksson et al. (1984). The models used by Lambert et al. (1986) in their abundance analyses for 30 bright $\mathrm{N}$ stars are plane parallel but otherwise fairly similar to those of Jørgensen et al. Opacity Sampling is used for all recent N star models.

Comparison of observations of spectra of these models with current temperature scales, partly based on angular diameters and partly on the Infrared flux method or colours, show problems with the polyatomic bands from $\mathrm{HCN}$ and $\mathrm{C}_{2} \mathrm{H}_{2}$ which come out too strong (Lambert et al. (1986), Jørgensen (1989)). Systematic revisions of the effective temperatures by about $200 \mathrm{~K}$ upwards would be needed to remedy this discrepancy. Another problem in the abundance analysis of Lambert et al. - based on high resolution IR FTS spectra - were the $\mathrm{CH}$ vibration-rotation lines being significantly weaker than calculated. This mismatch has not been understood and needs further exploration. E.g., an increase in effective temperature by about $500 \mathrm{~K}$ would be needed to remedy this. An astonishing fact was that the nitrogen abundances derived mainly from $\mathrm{CN}$ lines were only solar or less. Many consistency checks come, however, out positively in the analysis of Lambert et al.; among those were ${ }^{12} \mathrm{C} /{ }^{13} \mathrm{C}$ ratios in fair agreement from different criteria. These were, however, recently questioned by Ohnaka and Tsuji (1996) who got considerably lower values from lines in the crowded near-IR region. These authors suggest that severe differences between the model atmospheres used should be the explanation - this, however, does not seem to be the case. The major difference between the grids is that the Ohnaka and Tsuji models include a turbulent pressure different from zero, which the models of Lambert et al. do not. This, however, may be treated just as a gravity shift, which does not change the isotopic ratios significantly. We note that the effective temperatures of Ohnaka and Tsuji, based on the Infrared flux method, are several hundred $\mathrm{K}$ in excess of those of Lambert et al. and systematically higher than current angular diameter measurements. Further analyses of carbon star spectra, with systematic consistency checks using different criteria, must be pursued.

It is not known to which extent discrepancies like those mentioned above could be ascribed to departures from LTE. In fact, for the cool stars this is an almost totally unexplored area.

Dust probably plays an important role in all cool star atmospheres, as an opacity source and for the transfer of momentum and mass, as well. 
Future models must include this, which will require improvements in the theory of dust formation, and dynamics.

Velocity fields in the atmospheres of red giant stars show quite interesting structures, indicating the inadequacy of the standard micro/macroturbulence concepts. Important results indicating this were obtained by Tsuji (1991) in his study of CO VR lines in high-resolution spectra of M giants. Line shifts and bisectors reveal complicated velocity fields, possible to interpret as granular motions. (Especially, standard abundance determinations based on saturated lines, e.g., the 2.3 micron CO bands, may lead to systematic errors.) 3D simulations of convection should also be attempted for these stars (see further below). Also Mira models with a simultaneous detailed treatment of pulsation, radiation and dust formation should be developed. No doubt, a much closer interaction between detailed modelling and systematic observing will be needed for significant further progress in this field.

\section{Cool dwarfs: a field in rapid development}

A major step forward in the analysis of $M$ dwarfs is the work by France Allard, Peter Hauschildt, Hugh Jones, and collaborators. Allard and Hauschildt (1995) published an extensive grid for $M$ dwarfs with $T_{\text {eff }}$ down to $1500 \mathrm{~K}$, and different gravities and metallicities. A great number of molecular bands and atomic lines were handled in opacity sampling, however, the most important line opacities, water vapour and $\mathrm{TiO}$, were still only treated with rough statistical methods. These models represent a significant improvement in consistency, and in their fit to observed near-infrared spectra. However, severe discrepancies prevail in the infrared beyond $1.3 \mu \mathrm{m}$ which is seen both in low resolution spectra and in IR two-colour diagrams. This is probably mainly due to over-estimated water vapour absorption. The calculated spectra demonstrate a number of interesting and important properties which are partly counter-intuitive, e.g. the strengthened metal hydride absorption with decreasing metallicity. Moreover, the overall flux is not shifted very much towards longer wavelengths when the effective temperature is decreased, due to the increased water vapour absorption and, at cooler temperatures, the growing collision-induced absorption (CIA) by $\mathrm{H}_{2}$, centred around $2 \mu \mathrm{m}$.

Later, Jones et al. (1995) studied the behaviour of the $\mathrm{H}_{2} \mathrm{O}$ absorption bands in cool dwarfs around $3 \mu \mathrm{m}$ with similar models but with a more detailed line-by-line treatment of $\mathrm{TiO}$ and with data for $\mathrm{H}_{2} \mathrm{O}$ in the synthetic spectra based on recent ab-initio calculations. From matching the spectra the authors find effective temperatures in fair agreement with other determinations, however, with errors on the order of $7 \%$. The authors warn that 
more definite determinations must wait until the water-vapour opacity calculations are extended to wavelengths below $2 \mu \mathrm{m}$. A better understanding of molecular line broadening by damping is also needed - the results by Jones et al. (1995) may indicate that the damping constants have been overestimated when chosen according to prescriptions for atomic lines.

Jones et al. (1996) explored the capacity of the models of Allard and Hauschildt (1995) to reproduce $\mathrm{M}$ dwarf spectra in the $J$ band. The region studied contains strong KI, Fe I, Mg I lines and FeH bands. A reasonable, though not yet satisfactory, agreement is found - suggesting metallicities from -2.5 to +0.5 on a logarithmic scale relative to the Sun. The problems with the fit of the Fe I lines are at least partially due to too great $\log g f$ values.

The same models were also used by Leggett et al. (1996) in discussing infrared low-resolution spectra of $16 \mathrm{M}$ dwarfs (M0-M6.5). They found that the spectra give consistent effective temperature estimates, although discrepancies remain between theory and observation such as an excess model flux in $V$, problems with hydrides such as $\mathrm{FeH}$ and with reproducing IR fluxes to the $10 \%$ level. The effective temperatures derived are hotter than the commonly used temperature scale (by Berriman and Reid (1987)) would suggest by on average $130 \mathrm{~K}$. With these temperatures new radii were estimated. The $T_{\text {eff }}$ and radii thus obtained, as well as their dependence on metallicity, seem to agree well with most recent stellar models, which resolves a long-standing discrepancy. A relatively high $T_{\text {eff }}$ scale for earlier M-type dwarfs is also supported by the study, based on independent models, of Brett and Plez (1993).

Tsuji et al. (1996) (see also references therein) have explored the results of dust-formation in $M$ dwarf model atmospheres. The basic effect is a heating of the photosphere by several hundred Kelvin for stars with $T_{\text {eff }}<2500 \mathrm{~K}$, counteracting the dust condensation. This may keep the surface layers just at the condensation temperatures of the most abundant grain species. The effect on the spectrum is a general weakening of molecular bands, due to the stronger dust absorption and less molecules in the surface layers. The application of these dusty models in determining effective temperatures from spectra tend to favour a fairly low scale as compared with similar determinations with non-dusty models. For the interesting continuation of these studies to the very cool end, the brown dwarfs, see Baraffe's contribution in the present Proceedings.

The models for $M$ dwarfs are now advanced enough to be tried also in detailed abundance analyses. Recent instruments (such as the Kitt Peak Phoenix IR spectrometer) admit such work for many stars. Analyses of M dwarfs in binaries or other systems with hotter components with known compositions are important as tests. Further studies of the role of stellar 
activity for the structure of $\mathrm{M}$ star atmospheres must also be carried out.

\section{R CrB stars: progress and a worrying failure}

This interesting but relatively small group of stars, with their $\mathrm{H}$ poorness, carbon richness and sudden declines of brightness, have earlier only been analysed with un-blanketed model atmospheres. Those models did not produce both high-excitation features, like He I or C II lines, and low excitation features such as $\mathrm{C}_{2}$ lines which often are present in the same stellar spectra. We have calculated a grid of blanketed LTE models for these stars (Asplund et al. (1997)) and found that blanketing is so strong, since the continuous absorption is severely reduced by the reduction of hydrogen, that the temperature gradient is raised such that both the high- and low-excitation features show up at roughly the right strength. The strong temperature gradient and the He I absorption at depth even bring the stars across the effective Eddington instability border where radiative acceleration is taking over gravitation. Asplund and Gustafsson (1996) suggest this to be an important factor behind the sudden declines, ascribed to occultation by dust formed in gas clouds ejected from the stars.

However, when using the models in detailed abundance analyses we, in collaboration with David Lambert and Kameswar Rao, came across an interesting problem. The abundant CI lines in the stellar spectra are all considerably weaker than those predicted, corresponding to an abundance reduction by about a factor of 4 (Lambert et al. (1997), Gustafsson and Asplund (1996)). The discrepancy cannot be removed by a simple abundance change - the continuous opacity is also mainly due to C I bound-free absorption, originating from levels only slightly higher than those of the lower levels of the lines. Therefore, a temperature change is also not a realistic explanation, nor a gravity change or a change in microturbulence parameter. Non-LTE effects are also found to be small, and errors in $g f$ values or bound-free absorption coefficients are less probable. The most probable explanation is a drastic temperature structure modification, e.g. related to the unstable region in the deep atmosphere. Radiation-hydrodynamic simulations should be used to explore the stability and the structure of objects of this character, with a super-Eddington zone in the deep photosphere. However, it is worrying that this - in principle very straightforward and simple - test case of the classical model atmospheres come out with a great inconsistency. In other more normal stars this inconsistency would have been masked as an abundance effect. 


\section{Solar-type stars: home-work to do!}

A great step forward in stellar physics is the construction of reasonably realistic models of convection and granulation. Pioneering 3D convection models with a rather coarse spatial resolution and assuming an an-elastic fluid were constructed by Nordlund (1982), Nordlund and Dravins (1990), and compared with observations of spectral-line profiles by Dravins and Nordlund (1990a), (1990b). Compressible 2D models were made by Steffen and collaborators (Freytag et al. (1996), and references therein). (Magneto)hydrodynamic simulations of fully compressible convection can now be made in $3 \mathrm{D}$, with a relatively high spatial resolution and a rather detailed consideration of radiative transfer (Rast et al. (1993), Nordlund and Stein (1995), and references therein), a possibility not yet exploited for other stars than the Sun.

Attempts to fit the solar spectrum and limb-darkening with more classical flux-constant plane-parallel model atmospheres have given conflicting results. Gustafsson et al. (1975) found their blanketed solar model to be too bright in the ultraviolet by e.g. $25 \%$ around $350 \mathrm{~nm}$ and ascribed this to a missing opacity source, probably a veil of weak metal lines. Kurucz later, using a more extensive atomic line list found a good agreement (Kurucz (1992)) which was verified by Edvardsson et al. (1993) with essentially the same list but a different model atmosphere program (OSMARCS). (Note, however, the critical remarks made by Bell et al. (1994)). The Kurucz (1992) model (ATLAS9) and the OSMARCS model are different as regards prescriptions for convection. In particular, the ATLAS9 model has an ad hoc consideration of convective overshoot, which gives the temperature structure a characteristic hump around $\tau($ cont $)=0.6$ of about $200 \mathrm{~K}$, and an even more extended effect for metal-poor models (cf. Fig 11 in Gratton et al. (1996)). This difference in structure makes the ATLAS9 model reproduce solar limb-darkening observations better than the OSMARCS model (Blackwell et al. (1995)), but none of them are as successful as the classical Holweger-Müller empirical model in this respect.

The $\mathrm{H} \beta$ wings of the ATLAS9 model are, however, far too bright, and convective overshoot must be switched off and the mixing length parameter $\alpha$ lowered to 0.5 in order for a good agreement with observations to result (van't Veer-Menneret and Megessier (1996), Fuhrmann et al. (1993)). Similar results were found for Procyon (F5 IV-V). In OSMARCS models the standard choice of $\alpha=1.5$ leads to a good fit, which is since the value of the structure parameter $y$ in the mixing-length formalism (cf Henyey et al. (1965)) is chosen to 0.076 instead of 0.5 . Similar results were obtained by Fuhrmann et al. (1993), see also Fuhrmann et al. (1994), who stress the significance of the Balmer line profiles as tests of the model atmospheres.

Obviously, none of the two theoretical models give satisfactory results in 
both tests. This is presumably because the real Sun can not be represented by a flux-constant $1 \mathrm{D}$ model with mixing-length convection. It would be of great interest to check the predicted limb-darkening and spectrum, not the least that of the Balmer lines, of the Nordlund 3D models.

What models should then be used for solar-type stars? Gratton et al. (1996) showed that the differences between the OSMARCS models of Edvardsson et al. (1993) and the ATLAS9, as well as the more recent Kurucz models from 1995 with certain errors corrected, are very considerable, the latter being several hundred $\mathrm{K}$ hotter in the flux-forming layers due their "convective overshoot". This introduces a considerable uncertainty in the effective temperature scale. In a detailed study of the temperature scale for solar-type dwarfs, as derived from Balmer line profiles and various colour indices, Fuhrmann et al. (1994) found significant inconsistencies and scatter between different criteria which casts doubt on scales based on broad-band colours when used for individual stars as well as on calibrations by means of plane-parallel models in general. Gratton et al. (1996) also derived a semi-empirical temperature calibration of colours for metal poor late-type stars based on Kurucz ATLAS9 models. This calibration is significantly different from that of Edvardsson et al. (1993) and Nissen et al. (1994) which is based on calculated $(b-y)$ colours of OSMARCS models. The difference is ascribed by Gratton et al. to the differences in model structures - this requires further exploration. Gratton et al. check their scale by comparing calculated to observed $\mathrm{H} \alpha$ profiles - in view of the problems encountered with Kurucz's models, $\mathrm{H} \beta$ profiles should also be checked.

Obviously, the situation for metal-poor dwarf atmospheres is far from satisfactory - uncertainties by $400 \mathrm{~K}$ and 0.4 dex could be present in $T_{\text {eff }}$ and $[\mathrm{Fe} / \mathrm{H}]$, respectively! Some of the effect may be compensated for by the fact that convective overshoot will also affect the line forming atmospheric layers. For the line strengths of many lines, however, the temperature gradient is what matters and this is drastically different in the different models.

Studies based on spectra of $3 \mathrm{D}$ models of convection for solar-type dwarfs of different metallicities are thus urgently needed. Simulations of this type, combined with detailed spectroscopic observations, up along the sub-giant and red-giant branch are also important. Moreover, the advance in non-LTE blanketing already exploited for hotter stars should be applied for solar-type stars. Until this has been done one should systematically use weak lines, formed in layers close to the continuum forming layers, in abundance analyses and also explore the virtues of semi-empirical model atmospheres in detailed abundance analyses. There have been only scattered attempts to derive such models for late-type stars, from individual line profiles or other criteria (see Gustafsson and Jørgensen (1994) for a review; see also Morossi's presentation at this meeting). 
The solar-type stars are of key significance in the study of galactic evolution. Their ages may be determined and range all across the life-span of the Galaxy, and their rich visual spectra enable the determination of abundances which may be assumed to be characteristic of the gas from which the stars once formed. In fact, much of our understanding of the evolution of the Galaxy emanates from studies of these stars (cf., e.g., Edvardsson et al. (1993). They may also, with available techniques, be used as tracers of the chemical evolution in nearby dwarf galaxies (cf. Ardeberg et al. (1997)). Given this significance, it is astonishing that the great progress made in hydrodynamics and in non-LTE modelling, has hardly been exploited in analyses of solar-type stars. A particularly significant group of stars for which 3D simulations should now be explored (and compared with profiles of hydrogen, and other strong lines and bisectors of metal lines) and detailed non-LTE analyses ought to be made systematically, is the metal-poor dwarfs. In view of the significance of their abundances for cosmology and for the study of the evolution of the early Galaxy, this must be regarded a high-priority project.

\section{References}

Allard, F. and Hauschildt, P.H.. (1995) ApJ 445, 433

Allard, F., Laylor, L., Alexander, D.R., Hauschildt, P.H. (1994) BAAS 187, 10311

Anderson, L.S. (1989) ApJ 339, 558

Anderson, L. (1991), in Crivellari et al. (1991), p. 29

Ardeberg, A., Gustafsson, B., Linde, P., Nissen, P.E. (1997), A\&A, in press

Asplund, M. and Gustafsson, B. (1996) Hydrogen-deficient stars, C.S. Jeffery \& U. Heber (eds.), ASP Conf. Ser. Vol. 96, 39

Asplund, M., Gustafsson, B., Kiselman, D., Eriksson, K. (1997) A\&A 318, 521

Avrett, E.H. (1996) in Strassmeier \& Linsky (1966), p. 503

Bell, R.A., Paltoglou, G. and Tripicco, M.J. (1994) MNRAS 268, 771

Berriman, G.B. and Reid, N. (1987) MNRAS 227, 315

Bessell, M., Castelli, F. and Plez, B. (1997), in preparation

Blackwell, D.E., Lynas-Gray, A.E., Smith, G. (1995) A\&A 296, 217

Brett, J.M. and Plez, B. (1993) Proc. ASA 10, 250

Castelli, F. and Kurucz, R.F. (1994) A\&A 281, 817

Crivellari, L., Hubeny, I., Hummer, D.G. (eds.) (1991) Stellar Atmospheres: Beyond Classical Models, NATO ASI Series, Vol. 341

Dravins, D. and Nordlund, A. (1990a) A\&A 228, 184

Dravins, D. and Nordlund, $\AA$. (1990b) A\&A 228, 203

Dreizler, S. and Werner, K. (1993) A\&A 278, 199

Edvardsson, B., Andersen, J., Gustafsson, B. et al. (1993) A\&A 275, 101

Eriksson, K., Gustafsson, B., Jørgensen, U.G., Nordlund, A. (1984) A\&A 132, 37

Frandsen, S. (1974) A\&A 37, 139

Freytag, B., Ludwig, H.-G. and Steffen, M. (1996) A\&A 313, 497

Fuhrmann, K., Axer, M., Gehren, T. (1993) A\&A 271, 451

Fuhrmann, K., Axer, M., Gehren, T. (1994) A\&A 285, 585

Gabler, R., Gabler, A., Kudritzki, R.P., Puls, J. and Pauldrach, A. (1989) A\&A 226, 162

Gabler, R., Kudritzki, R.P. and Mendez, R.H. (1991) A\&A 245, 587

Gabler, R., Gabler, A., Kudritzki, R.P. and Mendez, R.H. (1992) A\&A 265, 656 
Gigas, D. (1986) A\&A 165, 170

Gigas, D. (1988) A\&A 192, 264

Gratton, R.G., Caretta, E., Castelli, F. (1996) A\&A 314, 191

Gulliver, A.F., Hill, G., Adelman, S.J. (1994) ApJ 429, L81

Gustafsson, B. (1995) Astrophysical Applications of Powerful New Databases, S.J. Adelman \& W.L. Wiese (eds.), ASP Conf. Ser. Vol. 78, 347

Gustafsson, B. and Asplund, M. (1996) Hydrogen-deficient stars, C.S. Jeffery \& U. Heber (eds.), ASP Conf. Ser. Vol. 96, 27

Gustafsson, B., Bell, R.A., Eriksson, K., Nordlund, A. (1975) A\&A 42, 407

Gustafsson, B. and Jørgensen, U.G. (1985) Calibration of Fundamental Stellar Quantities, Proc. IAU Symp. Vol. 111, D.S. Hayes et al. (eds), D. Reidel Publ. Co.,p. 303

Gustafsson, B. and Jørgensen, U.G. (1994) A\&AR 6, 19

Haas, S., Dreizler, S., Heber, U., Jeffery, S. and Werner, K. (1996) A\&A 311, 669

Hamann, W.-R. (1995) Wolf-Rayet Stars: Binaries, Colliding Winds, Evolution, Proc. IAU Symp. Vol. 163, K.A. van der Hucht, P.M. Williams (eds.), Kluwer, p. 105

Henyey, L., Vardya, M.S., Bodenheimer, P. (1965) ApJ 142, 841

Hillier, D.J. (1995) Wolf-Rayet Stars: Binaries, Colliding Winds, Evolution, Proc. IAU Symp. Vol. 163, K.A. van der Hucht, P.M. Williams (eds.), Kluwer, p. 116

Hubeny, I. and Lanz, T. (1995) A\&A 439, 875

Huenerth, G and Ulmschneider, P. (1995) A\&A 293, 166

Jørgensen, U.G. (1989) ApJ 344, 901

Jørgensen, U.G. (1997) Molecules in Astrophysics, E. van Dieshoeck (ed.), Kluwer Academic Publ., in press

Jørgensen, U.G., Johnson, H.R., Nordlund, A. (1992) A\&A 261, 263

Jones, H.R., Longmore, A.J., Allard, F. et al. (1995) MNRAS 277, 767

Jones, H.R., Longmore, A.J., Allard, F., Hauschildt, P.H. (1996) MNRAS 280, 77

Kurucz, R.L. (1992) Rev. Mex. Astron. Astrof. 23, 181

Kurucz, R.L. (1995) Astrophysical Applications of Powerful New Databases, S.J. Adelman \& W.L. Wiese (eds.), ASP Conf. Ser. Vol. 78, 205

Lambert, D.L., Gustafsson, B., Eriksson, K., Hinkle, K. (1986) ApJS 62, 673

Lambert, D.L., Rao, K.W., Asplund, M., Gustafsson, B. (1997), in preparation

Leggett, S.K., Allard, F., Berriman, G. et al. (1996) ApJS 104, 117

Lemke, M., Venn, K.A. (1994) A\&A 309, 558

Nissen, P.E., Gustafsson, B., Edvardsson, B., Gilmore, G. (1994) A\&A 285, 440

Nordlund, A. (1982) A\&A 107, 1

Nordlund, A. and Dravins, D. (1990) A\&A 228, 115

Nordlund, A. and Stein, R.F. (1995), in Stellar Evolution: What Should Be Done, Proc. 32nd Liege Int. Astroph. Coll, p. 75.

Ohnaka, K.. and Tsuji, T. (1996) A\&A 310, 933

Pauldrach, A., Feldmeier, A., Puls, J. and Kudritzki, R. (1993) Space Sci. Rev. 66, 105

Pauldrach, A., Kudritzki, R.P., Puls, J. et al. (1994) A\&A 283, 525

Piskunov, N.E., Kupka, F., Ryabchikova, T.A. et al. (1995) A\&AS 112, 525

Plez, B., Brett, J.M., Nordlund, A. (1992) A\&A 256, 551

Rast, M.P., Nordlund, Å., Stein, R. and Toomre, J. (1993) ApJ 408, L53

Rentzsch-Holm, I. (1996) A\&A, 305, 275

Schaerer, D., Schmutz, W. (1994) A\&A 288, 231

Scharmer, G.B. (1981) ApJ 249, 720

Seaton, M.J., Yu Yan, Mihalas, D. and Pradhan, A.K. (1994) MNRAS 266, 805

Strassmeier, K.G. and Linsky, J.L. (1996) Stellar Surface Structure, Proc. IAU Symp.

Vol. 176, Kluwer Academic Publ., p. 501

Takeda, Y. (1992) PASJ 44, 649

Tsuji, T. (1991) A\&A 245, 203

Tsuji, T., Ohnaka, K., Auki, W. (1996) A\&A 30, 1

van't Veer-Menneret, C., and Megessier, C. (1996) A\&A 309, 879 


\section{DISCUSSION}

MONIQUE SPITE: Is it possible now with the new MARCS models to have a good representation of the centre-to-limb variation of the solar lines?

BENGT GUSTAFSSON: Concerning spectral lines, I don't know yet. The limb darkening in the continuum is, however, still not very well reproduced (c.f. Blackwell et al., 1995, A\&A 314, 191).

MIKE BESSELL: What was the difference in the lithium abundance from the hydrodynamic simulation and the $\mathrm{p}-\mathrm{p}$ atmosphere?

BENGT GUSTAFSSON: For LTE models in the solar case it seems to be less than $0.1 \mathrm{dex}$. It could be worse in more metal-poor stars.

ROGER BELL: Since you showed the effect of including more and more $\mathrm{H}_{2} \mathrm{O}$ lines, is it necessary to include lines of $\mathrm{H}_{2} \mathrm{O}^{17}$ and $\mathrm{H}_{2} \mathrm{O}^{18}$ ?

BENGT GUSTAFSSON: Yes, it might be necessary for detailed models.

MIKE BESSELL: The problem with the $R$ Cor Bor stars may be related to that seen in other carbon-rich objects. The carbon-rich dwarf G77-61 has been determined to have a $\mathrm{Mg}$ abundance 100 times lower than expected from its space motion. Similarly, Lamers has analysed Pop I A-F supergiants in advanced evolutionary stages which are C-rich. He finds $\mathrm{Fe}$ abundances as low as $1 \%$ solar, although again, one would expect near solar abundances. It seems that in C-rich atmospheres metal lines are computed much too strong, as though an important opacity source is missing.

BENGT GUSTAFSSON: Yes, that is an interesting possibility. The question is what this opacity could be due to.

ROBERT KURUCZ: The convective models have systematic errors. The physical basis for the errors is understood. Here is how to proceed: make ad hoc corrections to the predictions of each grid; tabulate the systematic error in $T_{\text {eff }}$ as a function of $T_{\text {eff }}$ from methods using wings of $\mathrm{H} \alpha, \mathrm{H} \beta$, etc.; tabulate corrections to a $V$ magnitude as a function of $T_{\text {eff }}$; tabulate corrections to $\mathrm{UV}$ and visible flux; then determine which lines species are sensitive to convective structure and do not use them in abundance analyses.

BENGT GUSTAFSSON: I think one should put a strong emphasis on developing and trying as physically self-consistent approaches as possible before adopting ad-hoc procedures - essentially introducing extra free parameters.

CLAUDE MEGESSIER: Could you comment more on the problem of carbon in the $\mathrm{R}$ Cor Bor stars. Why do you think that NLTE would not improve the situation? The work by Hubeny and Lanz shows that the NLTE treatment of $C$ has a large effect on the $T(\tau)$ temperature law.

BENGT GUSTAFSSON: In the much cooler $\mathrm{R}$ Cor Bor stars (as compared with the white dwarfs in the work of Hubeny and Lanz), carbon has been found to be close to LTE by detailed statistical-equilibrium 
calculation by Asplund and Ryde at Uppsala. This was obtained for a given model atmosphere - I do not think, however, the coupling between the CI excitation/ionization and the model structure to be such that this situation would change considerably in a self-consistent model.

RAINER WEHRSE: There is another side concerning the progress caused by the introduction of the accelerated $\Lambda$ iteration: the accelerated $\Lambda$ iteration is simply a Jacobi iteration, which is well known in mathematics for more than 100 years and is considered obsolete for more than 30 years. There are now much more efficient methods available, as e.g., multi-grid or Krylow space methods.

BENGT GUSTAFSSON: Maybe, but in spite of this, the (re)invention of the ALI has really been a major step forward in this field of research. It is also, not the least in combination with the complete linearization as proposed by Hubeny and Lanz, a very flexible and efficient method. A comparison of disadvantages and advantages of that method with multi-grid and Krylow space methods would be interesting.

ROGER BELL: Where did you get your value of $3 \%$ for the C/He ratio?

BENGT GUSTAFSSON: We used values of $0.3,1$ and $3 \%$ for the $\mathrm{C} / \mathrm{He}$ ratio, mainly inspired by determinations for EHe stars, which we think are evolutionarily related to the $R$ CrB stars. At such high ratios, He mainly acts as an inert element in the $R \operatorname{CrB}$ photospheres. 